\title{
Probing Atomic Migration in Nanostructured Multilayers: Application of X-Ray Standing Wave Fields
}

\author{
S. Bera, ${ }^{1}$ K. Bhattacharjee, ${ }^{1}$ G. Kuri, ${ }^{2, \dagger}$ and B. N. Dev ${ }^{1,3, *}$ \\ ${ }^{1}$ Institute of Physics, Sachivalaya Marg, Bhubaneswar-751005, India \\ ${ }^{2}$ Hamburg Synchrotron Radiation Laboratory (HASYLAB) at DESY, Notkestrasse 85, D-22603 Hamburg, Germany \\ ${ }^{3}$ Indian Association for the Cultivation of Science, $2 A$ and $2 B$ Raja S. C. Mullick Road, Jadavpur, Kolkata 700032, India
}

(Received 13 January 2007; published 10 May 2007)

X-ray standing wave fields, excited in periodic nanostructured multilayers during Bragg diffraction, have been used to probe atomic migration in multilayers. Ion beam induced migration of Fe impurity atoms from the $\mathrm{C}$ layers to the $\mathrm{Pt}$ layers in a $\mathrm{Pt}(\mathrm{Fe}) / \mathrm{C}(\mathrm{Fe})$ multilayer, where each layer is about $2 \mathrm{~nm}$ thick, has been detected. With a depth resolution better than $0.2 \mathrm{~nm}$ of this technique, the direction of Fe migration (here outward) and the change of Fe concentration in C (also Pt) layers have been determined. The results of such measurements are important for understanding the properties of multilayers, for example, the evolution of ferromagnetism in the present example [Dev et al., Microelectron. Eng. 83, 1721 (2006)].

DOI: 10.1103/PhysRevLett.98.196103

Layered synthetic microstructures or multilayers consisting of thin layers of alternating elements or compounds have unique structural, mechanical, electronic, and magnetic properties with a wide range of applications [1]. New frontiers involving fields like magnetoelectronics and spin electronics (spintronics) are emerging where layered magnetic structures are being used. On the other hand, tailoring materials properties using energetic ion beams is well known. Ion beams have been used to fabricate modified materials for diverse electronic, optical, and tribological applications. Magnetic applications are also beginning to emerge. There have been recent efforts to understand interactions of energetic ions with nanostructured layered materials and the associated modification of their properties. For example, a spin-orientation transition was observed in $\mathrm{Co} / \mathrm{Pt}$ magnetic multilayers by ion irradiation [2] and its suitability for fabrication of patterned ultrahigh density recording media was pointed out. Ion irradiation has also been used to demonstrate fabrication of high density magnetic storage devices [3]. In magnetic multilayers where alternating layers are magnetic and nonmagnetic materials, some properties, such as magnetic coupling and magnetoresistance, can be changed by introducing some magnetic impurity atoms in the nonmagnetic layers and controlling their concentration [4]. Obviously, migration of such impurity atoms would change their concentration and spatial distribution and in turn the properties of the multilayers. Such atomic migration can be caused thermally, or otherwise, for example, by ion irradiation.

Here we consider ion beam induced atomic migration in a periodic multilayer where individual layers are about $2 \mathrm{~nm}$ thick. We demonstrate detection of not only atomic migration on a subnanometer length scale, but also the sense-outward or inward - of migration employing the $\mathrm{x}$-ray standing wave (XSW) technique. In a single crystal, $\mathrm{x}$-ray standing waves are generated when Bragg diffraction occurs. The standing wave pattern has the periodicity of the
PACS numbers: 68.49.Uv, 61.80.Jh, 68.65.Ac

corresponding diffraction planes (typically $0.2 \mathrm{~nm}$ ). In this case atomic displacements of typically one-hundredth of this distance (i.e., $2 \mathrm{pm}$ ) can be detected; whether this displacement is inward or outward can also be determined [5]. The XSW technique has been extensively used in condensed matter physics, especially in surface science [6]. In a periodic multilayer, x-ray standing waves are also generated when Bragg diffraction occurs from the multilayer [7]. For a typical case, where the multilayer period is $2 \mathrm{~nm}$, detection of atomic displacement or migration, outward or inward, with a precision of about onehundredth of this value (i.e., $20 \mathrm{pm}$ ) is possible. We use this high precision technique to detect ion irradiation induced migration of $\mathrm{Fe}$ in a $\mathrm{Pt} / \mathrm{C}$ multilayer containing $\mathrm{Fe}$ impurities [denoted hereafter by $\mathrm{Pt}(\mathrm{Fe}) / \mathrm{C}(\mathrm{Fe})]$. Although $\mathrm{Fe}$ is almost uniformly distributed over the whole depth of the multilayer in the virgin sample, we find that upon ion irradiation there is a surface-directed outward migration of $\mathrm{Fe}$ atoms, which prefer to leave $\mathrm{C}$ layers and end up in $\mathrm{Pt}$ layers. Gradual depletion of Fe from the $\mathrm{C}$ layers with increasing ion fluence over a depth scale of the multilayer period (here $\sim 4 \mathrm{~nm}$ ) was unambiguously determined by the XSW technique. We explain the reason for the outward migration of $\mathrm{Fe}$, evolution of $\mathrm{Fe}$ concentration with ion fluence, and the consequences of this atomic migration.

$\mathrm{Pt} / \mathrm{C}$ multilayer samples were prepared by alternate deposition of $\mathrm{Pt}$ and $\mathrm{C}$ layers on float glass substrates, by ion sputtering at a low Argon pressure of $0.1 \mathrm{mbar}$. A small amount (about 15 at. \%) [8] of Fe was introduced in the multilayers during growth. The sample specifications are $N=15$ (the number of layer pairs in the multilayer stack), $d=4.2 \mathrm{~nm}$ (multilayer period, i.e., the thickness of a Pt/C layer pair), $\Gamma=0.38$ (ratio of Pt layer thickness to $d$ ). The total thickness of the multilayer stack is about $63 \mathrm{~nm}$. Different parts of a large sample $\left(70 \times 30 \mathrm{~mm}^{2}\right)$ were irradiated with $2 \mathrm{MeV} \mathrm{Au}{ }^{2+}$ ions by rastering the ion beam on $30 \times 5 \mathrm{~mm}^{2}$ strips at various fluences (ions $/ \mathrm{cm}^{2}$ ) $[V$ (vir- 
gin), $B\left(1 \times 10^{14}\right), C\left(3 \times 10^{14}\right), D\left(5 \times 10^{14}\right), E(7 \times$ $\left.\left.10^{14}\right), F\left(1 \times 10^{15}\right)\right]$ at Institute of Physics, Bhubaneswar. The Au ions are implanted deep into the glass substrate having passed through the multilayer stack, leaving only ion beam induced effects in the multilayer. X-ray standing wave experiments were carried out at HASYLAB at DESY, Hamburg, at the ROEMO-I beam line using $14.0 \mathrm{keV}$ monochromatized $\mathrm{x}$ rays from a $\mathrm{Ge}(111)$ double-crystal monochromator. Pt $L$ and $\mathrm{Fe}-K$ fluorescent photons, excited by $14 \mathrm{keV}$ incident photons, were detected by a $\mathrm{Si}(\mathrm{Li})$ detector that was placed at an angle of $15^{\circ}$ with respect to the sample surface, and the reflectivity was measured by a $\mathrm{NaI}$ detector. The experimental set up is schematically shown latter in Fig. 3(a).

Here we follow the method of analysis as given in Refs. $[9,10]$. We first explain how outward or inward migration of Fe can be detected. Figure 1(a) schematically shows the formation of standing wave patterns while a first order Bragg diffraction of $\mathrm{x}$ rays occurs from a periodic multilayer. At the rising edge of the Bragg peak the antinodes of the standing wave are in the $\mathrm{C}$ layers. As the grazing angle of incidence advances through the Bragg peak the standing wave pattern moves inward finally moving by half the multilayer period $(d)$ at the falling edge of the Bragg peak, where the antinodes coincide with the Pt layers [9]. Referring to the inset of Fig. 1(b) let us assume that there is one atomic layer of $\mathrm{Fe}$ at the center of the $\mathrm{C}$ layer. This $\mathrm{Fe}$ layer is exposed to the maximum $\mathrm{x}$-ray intensity (antinode) when the angle of incidence corresponds to the rising edge of the Bragg peak, while at the falling edge of the Bragg peak the Fe layer is exposed to the minimum of $x$-ray intensity (node). As the intensity of emission of $\mathrm{Fe}-K \alpha(6.4 \mathrm{keV})$ fluorescence is proportional to the intensity of $\mathrm{x}$ rays $(14 \mathrm{keV})$ the $\mathrm{Fe}$ atoms are exposed to, the angular variation of the Fe- $K \alpha$ fluorescence yield will be given by the curve marked 0.5 in Fig. 1(b). If the Fe layer migrates inward, say to positions $0.4,0.3,0.2,0.1$, and 0 (the middle of the Pt layer), the Fe- $K \alpha$ fluorescence yield curve would change accordingly, as marked. The case of outward movement of $\mathrm{Fe}$ is illustrated in Fig. 1(c). In both cases the final position of the Fe layer is in the middle of a Pt layer ( 0 or 1$)$, and the two curves [" 0 " in Fig. 1(b) and "1" in Fig. 1(c)] are nearly the same except for the small depth-dependent difference due to absorption of incident as well as emitted $(\mathrm{Fe}-K \alpha)$ photons. We note that the way the shape of the curves changes from position 0.5 for inward [Fig. 1(b)] and outward [Fig. 1(c)] migration of Fe is quite different. Thus a measurement of the evolution of the Fe- $K \alpha$ fluorescence yield curve can distinguish between inward and outward migration of Fe.

We have already noticed a small difference between the curves 0 and 1 (with a depth difference of $\sim 4 \mathrm{~nm}$ ), which is an effect of $\mathrm{x}$-ray absorption. That is, absorption of $\mathrm{x}$ rays (both incident and fluorescent) in the multilayer affects the shape of the curve. When the effect of absorption for the whole multilayer ( 15 layer pairs) is taken into account the curves in Fig. 2 are generated. One can still distinguish

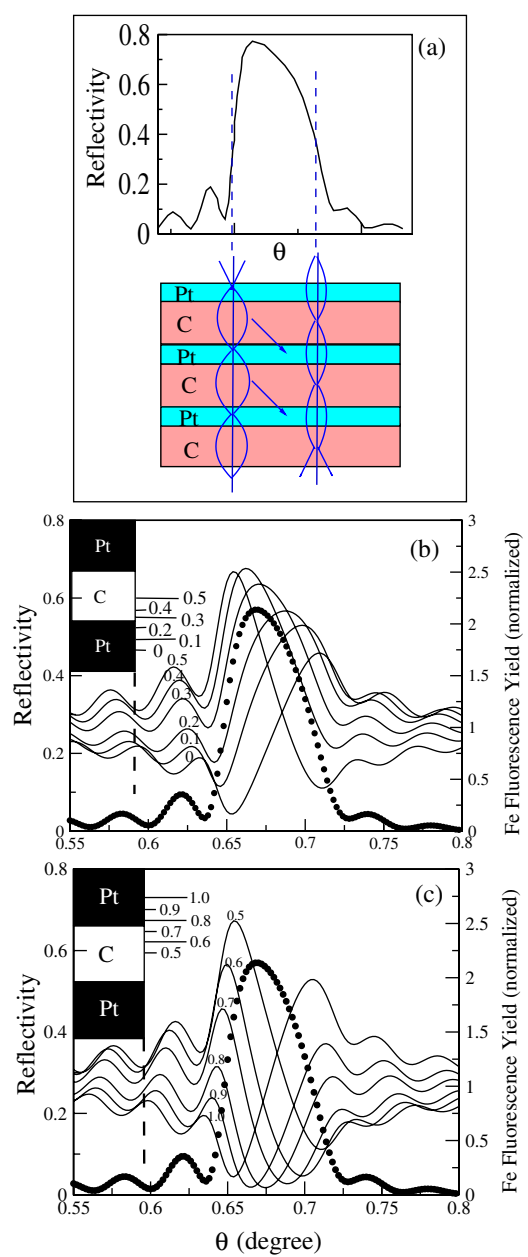

FIG. 1 (color online). (a) Illustration of the formation of x-ray standing wave patterns in a periodic multilayer during the first order Bragg diffraction from the multilayer. (b) Variation of $\mathrm{Fe}$ $K \alpha$ fluorescence yield curve when an atomic layer of $\mathrm{Fe}$ migrates from position 0.5 (center of a $\mathrm{C}$ layer) to $0.4,0.3, \ldots, 0.0$ (center of a Pt layer), etc. (inward) and (c) to $0.6,0.7, \ldots, 1.0$ (center of a Pt layer), etc. (outward). (See text for details.)

between inward and outward migration. The extent of modification of the shape of these curves (with respect to Fig. 1) depends on the exit angle of the fluorescent $x$ rays. The calculations are for an exit angle of $15^{\circ}$.

The difference between the Fe fluorescence yield curves in Figs. 1 and 2 is as follows. Let us explain it for one position, say, for position 0.5. In Fig. 1 the contribution of Fe from the middle (position 0.5) of only the topmost $\mathrm{C}$ layer is considered, while in Fig. 2 the total contribution of Fe from the middle of all $\mathrm{C}$ layers in the multilayer has been taken into account. Contributions from deeper layers are more affected by the attenuation effect. This is the reason for the differences in the curve shapes in Figs. 1 and 2.

Results of our experiment are presented in Fig. 3. The shape of the Pt- $L$ fluorescence yield curve [11] would be similar to curves 0 or 1 in Fig. 2, with small modifications due to ion irradiation effect (cf. Refs. [9,10]). This is what we observe in Fig. 3. For the Fe- $K \alpha$ fluorescence yield 

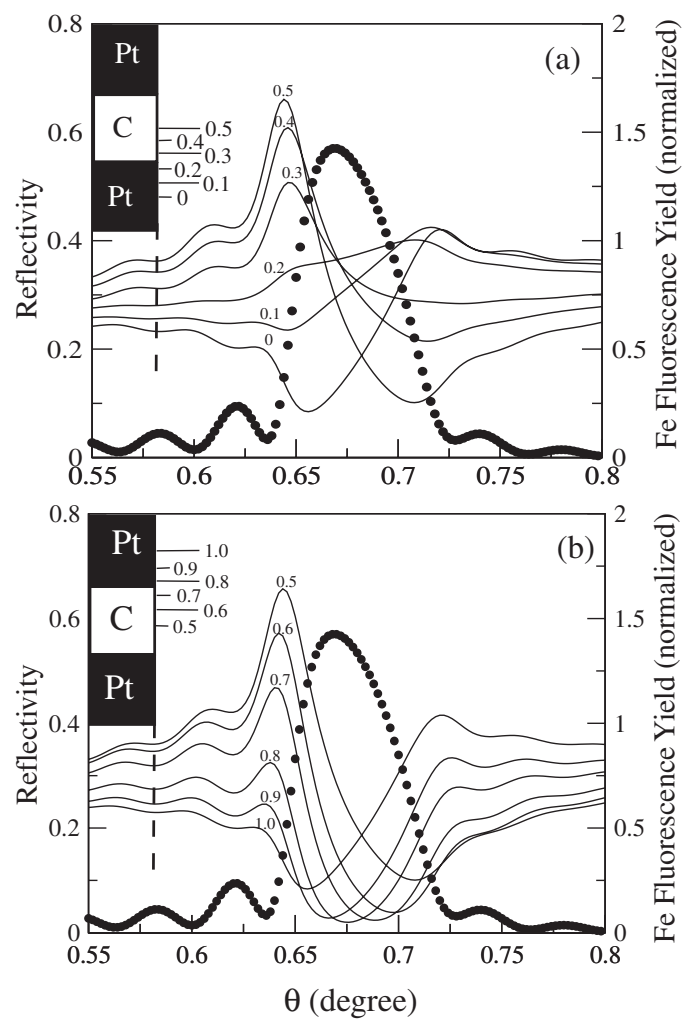

FIG. 2. When x-ray absorption effect for the whole multilayer stack is included, the curves in Figs. 1(b) and 1(c) give the modified curves (a) and (b), respectively. Following whether the fluorescence yield curve evolves like those in (a) or in (b), still inward and outward migration are quite distinguishable. (See text for details.)

curves we can follow the evolution from samples $V$ to $F$ [12]. They follow the evolution of shapes similar to those in Fig. 2(b) conforming to outward movement of Fe atoms. [The inward migration can be easily ruled out as the profiles never move through the shapes marked 0.3 or 0.2 in Fig. 2(a).] The fact that finally the Fe- $K \alpha$ fluorescence yield curve (for sample $F$ ) nearly coincides with that for Pt- $L$ fluorescence indicates the dominant presence of $\mathrm{Fe}$ in the Pt layers. Our detailed analysis, assuming a uniform depth distribution of $\mathrm{Fe}$ atoms in Pt layers and another uniform depth distribution of $\mathrm{Fe}$ in $\mathrm{C}$ layers, shows that $45 \%$ of all $\mathrm{Fe}$ atoms in the multilayer sample are in the $\mathrm{C}$ layers [13] in the virgin $(V)$ sample. This gradually reduces to $41 \%, 25 \%, 18 \%$, and $12 \%$ in samples $B, C, D$, and $F$, respectively. In the virgin $(V)$ sample, the remaining 55\% of all Fe atoms are initially present in the Pt layers. The Pt layers are enriched with $\mathrm{Fe}$ atoms upon ion irradiation, with $88 \%$ of all $\mathrm{Fe}$ atoms being present in the irradiated sample $F$. The change in the normalized Fe concentration in the $\mathrm{C}$ layers with ion fluence in irradiation is shown in Fig. 4. This dependence can be described well by

$$
C(\phi)=C(0) \exp (-\sigma \phi)
$$

where $\phi$ is the ion fluence (ions $\left./ \mathrm{cm}^{2}\right), C(0)$ is the $\mathrm{Fe}$ concentration in the virgin sample, $C(\phi)$ is the Fe concen-
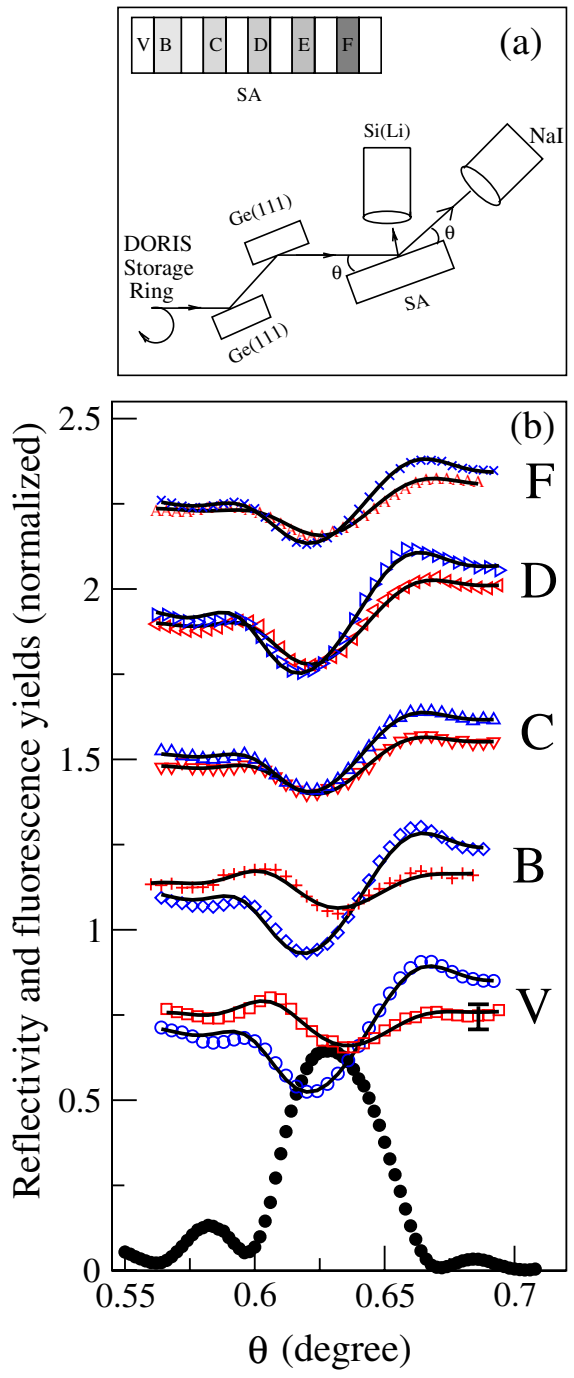

FIG. 3 (color online). (a) The experimental setup for the x-ray reflectivity and standing wave measurements and the sample (SA) are schematically shown. (b) First order Bragg peak from the virgin sample (solid black circle) and Pt $L \alpha$ [dark gray (blue) symbols: $V$, open circle; $B$, diamond; $C$, triangle up; $D$, triangle right; $F$, cross] and Fe $K \alpha$ [light gray (red) symbols: $V$, square; $B$, plus; $C$, triangle down; $D$, triangle left; $F$, character A] fluorescence yields from the virgin and the irradiated samples along with the fitted curves. Pt and Fe fluorescence yields from the irradiated samples are shifted vertically for clarity.

tration after irradiation at a fluence $\phi$, and $\sigma$ may be called ion beam induced atomic migration cross section. $\sigma$ is a parameter determined by the material and the kind of the ion beam and ion energy. The parameters obtained by fitting the data in Fig. 4 to Eq. (1) are $C(0)=1.00 \pm$ 0.05 and $\sigma=(1.6 \pm 0.2) \times 10^{-15} \mathrm{~cm}^{2}$.

This migration of $\mathrm{Fe}$ atoms into Pt layers gives rise to formation of FePt alloy particles giving rise to magnetism in the ion-irradiated multilayers $[14,15]$. The increase in $\mathrm{Fe}$ concentration in the Pt layers with increasing ion fluence also gives rise to increased magnetic strength in these ionirradiated multilayers as reflected in increasing coercive field in the hysteresis loop in Kerr rotation [15]. 


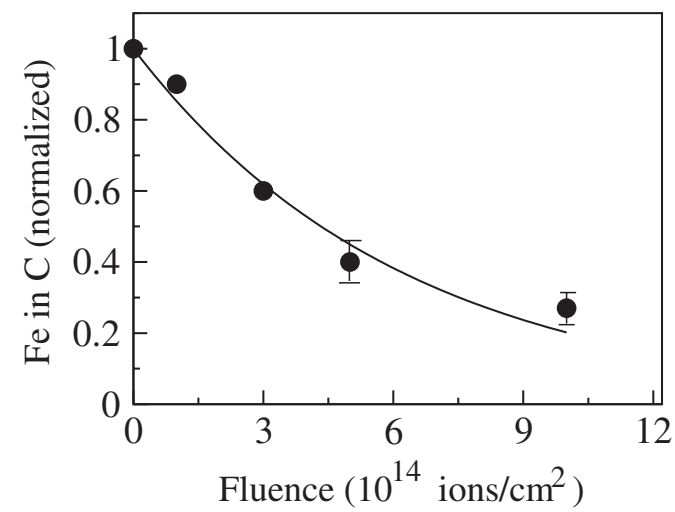

FIG. 4. The change in the normalized Fe concentration in the $\mathrm{C}$ layers with ion fluence is shown.

Why is the migration of Fe directed toward the surface (outward) of the multilayer? For binary alloys the prediction of surface segregation is based on the value of heat of segregation $(Q)$. If $Q$ is large and positive then segregation should occur. For the $\mathrm{Pt}(\mathrm{Fe})$ [solvent(solute)] system, Burton and co-workers failed to find any evidence of segregation of $\mathrm{Fe}$ to the surface of polycrystalline Pt by annealing the samples up to $1200^{\circ} \mathrm{C}$ under ultrahigh vacuum condition [16]. The predicted heat of segregation of Fe to the surface of $\mathrm{Pt}$ is below $2 \mathrm{kcal} / \mathrm{mole}$ [17]. This low value of $Q$ was their explanation for the absence of $\mathrm{Fe}$ surface segregation in Pt. But when they exposed their sample to a poorer vacuum $-1 \times 10^{-7}$ Torr of $\mathrm{O}_{2}$ - at $700{ }^{\circ} \mathrm{C}$, they observed surface segregation of $\mathrm{Fe}$ because $\mathrm{O}_{2}$ can draw $\mathrm{Fe}$ to the surface of $\mathrm{Pt}[16]$. In the present case during ion irradiation of our samples, the pressure in the irradiation chamber was about $1 \times 10^{-7}$ Torr. We believe the presence of $\mathrm{O}_{2}$ in the chamber at this pressure was responsible for dragging Fe toward the surface of Pt. Once this segregation starts, $\mathrm{Fe}$ atoms migrate in the outward direction throughout the multilayer stack.

In conclusion, we have demonstrated that atomic displacements or migration over subnanometer depth scale as well as the sense of displacement or migration (outward or inward) in nanostructured multilayers can be detected using x-ray standing wave fields in periodic multilayers, generated simply by Bragg diffraction from the multilayer. Such measurements using XSW will help understand the evolution of multilayer properties associated with atomic displacements or migration as they help understand the evolution of ferromagnetism in the present system as reported in Ref. [15]. Understanding atomic migration at nanometer and subnanometer length scales would provide the capability to tune multilayer properties.

We thank Professor K. Yamashita for providing the virgin $\mathrm{Pt}(\mathrm{Fe}) / \mathrm{C}(\mathrm{Fe})$ multilayer sample and Dr. D. K. Goswami for help during irradiation. The support from HASYLAB during experiments is gratefully acknowledged.
*Email address: bhupen@iopb.res.in; msbnd@iacs.res.in ${ }^{\dagger}$ Present address: LWV, NES, Paul Scherrer Institute, 5232 Villigen PSI, Switzerland.

[1] See, for example, Synthetic Modulated Structure, edited by L. L. Chang and B. C. Giesser (Academic, New York, 1985).

[2] D. Weller et al., J. Appl. Phys. 87, 5768 (2000).

[3] A. Dietzel et al., Adv. Mater. 15, 1152 (2003).

[4] S. S. P. Parkin et al., Europhys. Lett. 24, 71 (1993).

[5] S. K. Andersen, J. A. Golovchenko, and G. Mair, Phys. Rev. Lett. 37, 1141 (1976).

[6] J. Zegenhagen, Surf. Sci. Rep. 18, 199 (1993).

[7] T. W. Barbee and W. K. Warburton, Jr., Mater. Lett. 3, 17 (1984).

[8] Atomic concentration of $\mathrm{Fe}$ in the virgin sample has been determined from the relative intensities of $\mathrm{Fe}-K \alpha$ and $\mathrm{Pt}-L \alpha$ fluorescence peaks. This estimation has taken into account photoionization cross section for Fe $K$ shell and $\mathrm{Pt}$ $L_{\text {III }}$ subshell, their corresponding fluorescence yield, attenuation coefficients of incident photons and outgoing Fe- $K \alpha$ and Pt- $L \alpha$ photons in the multilayer, and an integration over the whole depth of the multilayer. This provides a ratio of atomic concentrations of 39:61 for Fe:Pt. However, the Fe atoms are almost uniformly distributed over the whole depth of the multilayer and a fraction of the $\mathrm{Fe}$ atoms is present in $\mathrm{C}$ layers. For a $\mathrm{Pt} / \mathrm{C}$ multilayer with $d=4.2 \mathrm{~nm}$ and $\Gamma=0.38$ (i.e., $1.6 \mathrm{~nm} \mathrm{Pt}$ layers, $2.6 \mathrm{~nm} \mathrm{C}$ layers: intended growth parameters) the ratio of the total number of $\mathrm{Fe}$ atoms $/ \mathrm{cm}^{2}$ to the sum of $\mathrm{Fe}, \mathrm{Pt}$, and $\mathrm{C}$ atoms $/ \mathrm{cm}^{2}$ turns out to be about 0.15 . Thus the average concentration of $\mathrm{Fe}$ is about 15 at. \%.

[9] S. K. Ghose and B.N. Dev, Phys. Rev. B 63, 245409 (2001).

[10] S. K. Ghose, D. K. Goswami, B. Rout, B. N. Dev, G. Kuri, and G. Materlik, Appl. Phys. Lett. 79, 467 (2001).

[11] The experimental curve is the effective shape of the Pt- $L$ fluorescence yield arising from $\mathrm{Pt}$ in the whole multilayer stack. Contribution from the thickness of the Pt layers has been taken into account for fitting the data following Ref. [9].

[12] The actual positions of the Bragg peaks are slightly different for the irradiated samples as in Ref. [10]. In this plot, for simplicity, we have aligned them at the same position (though these peaks are not shown) and plotted the fluorescence yield curves accordingly.

[13] The measured multilayer period is $d=4.2 \mathrm{~nm}$ where the thickness of $\mathrm{Pt}$ and $\mathrm{C}$ layers is $2.0 \mathrm{~nm}$ and $2.2 \mathrm{~nm}$, respectively. Using atomic densities of $\mathrm{Pt}$ $\left(6.6 \times 10^{22}\right.$ atoms $\left./ \mathrm{cm}^{3}\right)$ and amorphous $\mathrm{C}(1.0 \times$ $10^{23}$ atoms $/ \mathrm{cm}^{3}$ ), $45 \%$ of total $\mathrm{Fe}$ in $\mathrm{C}$ layers and $55 \%$ in Pt layers lead to average layer compositions of $\mathrm{Fe}_{15} \mathrm{C}_{85}$ and $\mathrm{Fe}_{26} \mathrm{Pt}_{74}$, respectively.

[14] S. Bera, B. Satpati, D. K. Goswami, K. Bhattacharjee, P. V. Satyam, and B. N. Dev, J. Appl. Phys. 99, 074301 (2006).

[15] B. N. Dev et al., Microelectron. Eng. 83, 1721 (2006).

[16] J. J. Burton and R. S. Polizzotti, Surf. Sci. 66, 1 (1977).

[17] J. J. Burton and E. S. Machlin, Phys. Rev. Lett. 37, 1433 (1976). 